\title{
STXBP1 promotes Weibel-Palade body exocytosis through its interaction with the Rab27A effector Slp4-a
}

\author{
Dorothee van Breevoort, ${ }^{1}$ Ambrosius P. Snijders, ${ }^{2}$ Nicola Hellen, ${ }^{3,4}$ Sarah Weckhuysen, ${ }^{5}$ Kathinka W. E. M. van Hooren, ${ }^{1}$ \\ Jeroen Eikenboom, ${ }^{6}$ Karine Valentijn, ${ }^{7}$ Mar Fernandez-Borja, ${ }^{8}$ Berten Ceulemans, ${ }^{9}$ Peter De Jonghe, ${ }^{5}$ Jan Voorberg, ${ }^{1}$ \\ Matthew Hannah, ${ }^{3,10}$ Tom Carter, ${ }^{3,11}$ and Ruben Bierings ${ }^{1,3}$

\begin{abstract}
${ }^{1}$ Plasma Proteins, Sanquin-Academisch Medisch Centrum Landsteiner Laboratory, Amsterdam, The Netherlands; ${ }^{2}$ Biomolecular Mass Spectrometry and Proteomics, Medical Research Council Clinical Sciences Centre, London, United Kingdom; ${ }^{3}$ Physical Biochemistry, Medical Research Council National Institute for Medical Research, London, United Kingdom; ${ }^{4}$ Myocardial Function, National Heart and Lung Institute, Imperial College, London, United Kingdom; ${ }^{5}$ Molecular Genetics, University of Antwerp, Belgium; ${ }^{6}$ Thrombosis and Hemostasis, Einthoven Laboratory for Experimental Vascular Medicine, Leiden University Medical Center, Leiden, The Netherlands; ${ }^{7}$ Molecular Cell Biology, Leiden University Medical Center, Leiden, The Netherlands; ${ }^{8}$ Molecular Cell Biology, Sanquin-Academisch Medisch Centrum Landsteiner Laboratory, Amsterdam, The Netherlands; ${ }^{9}$ Pediatric Neurology, University Hospital, University of Antwerp, Belgium; ${ }^{10}$ Public Health England, Colindale, London, United Kingdom; and ${ }^{11}$ Division of Biomedical Sciences, St. Georges,
\end{abstract} \\ University of London, United Kingdom
}

\section{Key Points}

- Recruitment of STXBP1 by Slp4-a promotes WeibelPalade body exocytosis.

- Ex vivo EIEE4 endothelial cells haploinsufficient for STXBP1 have impaired Weibel-Palade body exocytosis.
Vascular endothelial cells contain unique rod-shaped secretory organelles, called WeibelPalade bodies (WPBs), which contain the hemostatic protein von Willebrand factor (VWF) and a cocktail of angiogenic and inflammatory mediators. We have shown that the Rab27A effector synaptotagmin-like protein 4-a (SIp4-a) plays a critical role in regulating hormoneevoked WPB exocytosis. Using a nonbiased proteomic screen for targets for SIp4-a, we now identify syntaxin-binding protein 1 (STXBP1) and syntaxin-2 and -3 as endogenous SIp4-a binding partners in endothelial cells. Coimmunoprecipitations showed that STXBP1 interacts with syntaxin-2 and -3 , but not with syntaxin-4. Small interfering RNA-mediated silencing of STXBP1 expression impaired histamine- and forskolin-induced VWF secretion. To further substantiate the role of STXBP1, we isolated blood outgrowth endothelial cells (BOECs) from an early infantile epileptic encephalopathy type 4 (EIEE4) patient carrying a de novo mutation in STXBP1. STXBP1-haploinsufficient EIEE4 BOECs contained similar numbers of morphologically normal WPBs compared with control BOECs of healthy donors; however, EIEE4 BOECs displayed significantly impaired histamine- and forskolin-stimulated VWF secretion. Based on these findings, we propose that the Rab27A-SIp4a complex on WPB promotes exocytosis through an interaction with STXBP1, thereby controlling the release of vaso-active substances in the vasculature. (Blood. 2014;123(20):3185-3194)

\section{Introduction}

Endothelial cells line the lumen of all blood vessels, providing a highly dynamic barrier that plays a crucial role in maintaining vascular homeostasis. They contain specialized secretory organelles called Weibel-Palade bodies (WPBs) that allow the endothelium to store and release, in a regulated fashion, a presynthesized cocktail of hemostatic, inflammatory, and angiogenic mediators in response to endothelial activation, injury, or stress. ${ }^{1-3}$ The main component of these organelles is von Willebrand factor (VWF), a multimeric glycoprotein crucial for platelet plug formation and stabilizing coagulation factor VIII. In addition to VWF, several soluble chemokines (eg, interleukin-6 [IL-6], IL-8) as well as the integral membrane proteins CD63 and P-selectin are stored in these organelles. ${ }^{4-9}$ Coordinated expression of CD63 and P-selectin on the endothelial cell surface after WPB exocytosis is crucial for leukocyte extravasation at sites of inflammation. ${ }^{10}$ The presence of angiopoietin-2 and insulin-like growth factor-binding protein 7 in
WPBs points toward a critical role for the organelle in regulation of angiogenesis. ${ }^{11-13}$

The precise composition of mediators stored in WPBs depends crucially on the physical, mechanical, and chemical signals in the local microenvironment; for example, targeting of eotaxin-3, IL-8, and IL- 6 has been observed in response to pro-inflammatory mediators such as IL- $1 \beta$ or IL- $4,{ }^{5,7,14,15}$ whereas the inclusion of angiopoietin-2 is modulated under conditions that mimic shear stress. $^{16}$

Exocytosis of WPBs is triggered by a wide range of physiological secretagogues that trigger $\mathrm{Ca}^{2+}$ - and cyclic adenosine monophosphate (cAMP)-dependent signaling pathways, such as histamine and thrombin or vasopressin and epinephrine, respectively. ${ }^{3,17-20}$ Several key regulators of WPB exocytosis have been identified, including the small guanosine triphosphates (GTPases) RalA, Rab3A, Rab3D, and Rab27A. ${ }^{21-25}$ Rab27A plays a particularly crucial role in
Submitted October 31, 2013; accepted March 24, 2014. Prepublished online as Blood First Edition paper, April 3, 2014; DOI 10.1182/blood-2013-10535831 .

The online version of this article contains a data supplement.
The publication costs of this article were defrayed in part by page charge payment. Therefore, and solely to indicate this fact, this article is hereby marked "advertisement" in accordance with 18 USC section 1734. 
regulating WPB exocytosis through its ability to recruit multiple effector molecules, ${ }^{26}$ including myosin Va-Rab interacting protein (MyRIP), synaptotagmin-like protein 4-a (Slp4-a), and Munc13-4. MyRIP-dependent recruitment of the actin motor protein myosin $\mathrm{Va}$ to the WPB is proposed to link the organelle to the actin cytoskeleton, preventing access to and fusion with the plasma membrane. ${ }^{27,28}$ In contrast, both Slp4-a and Munc13-4 promote WPB exocytosis, ${ }^{25,29}$ although the mechanism remains unclear.

To investigate the mechanism by which Slp4-a regulates WPB exocytosis, we used an unbiased mass spectrometry approach aimed at identifying endogenous Slp4-a interactors in human endothelial cells. Using this screen, we identified syntaxin binding protein 1 (STXBP1, also known as Munc18-1) and syntaxin-2 and -3 as potential effectors of Slp4-a. RNA interference-mediated depletion of STXBP1 significantly impaired WPB release. Loss-of-function mutations in STXBP1 have been shown to be the cause of early infantile epileptic encephalopathy type 4 (EIEE4), a severe epileptic disorder. ${ }^{30-32}$ Blood outgrowth endothelial cells (BOECs), isolated from peripheral blood of a patient with EIEE4 carrying a de novo mutation in $S T X B P 1$, displayed impaired stimulated WPB release, providing further support for a role for STXBP1 in regulating VWF secretion. Our findings provide a link between the Rab27A-Slp4a complex on WPBs and a regulatory component of the Soluble NSF Attachment Protein REceptor (SNARE) complex, which controls the release of vaso-active substances in the vasculature.

\section{Methods}

\section{Antibodies and reagents}

Histamine, forskolin, 3-isobutyl-1-methylxanthine (IBMX), and ionomycin were from Sigma-Aldrich (Steinheim, Germany). Glutathione-Sepharose 4B was from GE Healthcare Europe GmbH (Diegem, Belgium). Fetal calf serum (FCS) was from Bodinco (Alkmaar, The Netherlands). Trypsin, Fura-2/AM, and Alexa 488-, Alexa 568-, and Alexa 633-conjugated secondary antibodies were from Invitrogen (Breda, The Netherlands). Primary antibodies used in this study are shown in supplemental Table 1 on the Blood Web site. DNA constructs are described in the supplemental data.

\section{Cell culture, BOEC isolation, and secretion assay}

Primary human umbilical vein endothelial cells (HUVECs) were obtained from Promocell (Heidelberg, Germany) and were grown in EGM-2 medium (Lonza, Basel, Switzerland) supplemented with $18 \%$ FCS (referred to henceforth as EGM-18) or as described. ${ }^{5}$ HEK293T cells were obtained from ATCC (Wessel, Germany) and were grown in Dulbecco's modified Eagle medium containing D-glucose, L-glutamine, and pyruvate (Life Technologies, Bleiswijk, The Netherlands) supplemented with $10 \%$ FCS, $100 \mathrm{U} / \mathrm{mL}$ penicillin, and $100 \mu \mathrm{g} / \mathrm{mL}$ streptomycin. BOECs were isolated as previously described. ${ }^{33}$ Fifteen milliliters of venous blood was drawn from 1 individual with EIEE4 and a de novo STXBP1 mutation (patient 4 in Deprez et $\mathrm{al}^{30}$ ) after the patient's parents signed an informed consent form for participation. The study was approved by the Sanquin Ethical Advisory Board and the Commission for Medical Ethics of the University of Antwerp. The study was conducted in accordance with the Declaration of Helsinki. Cells were stimulated with serum-free (SF) medium or SF medium containing $100 \mu \mathrm{M}$ histamine or $10 \mu \mathrm{M}$ forskolin supplemented with $100 \mu \mathrm{M}$ IBMX. VWF secretion was assayed by enzyme-linked immunosorbent assay (ELISA) as described previously. ${ }^{19} \mathrm{VWF}$ antigen levels in patient plasma were determined using ELISA.

\section{RNA interference}

Downregulation of endogenous expression of STXBP1 was performed essentially as described. ${ }^{29}$ Pools of 4 small interfering RNA (siRNA) oligo duplexes (ON-TARGETplus SMARTpool; Thermo Scientific, Rockford, IL) were used to deplete HUVEC of STXBP1 (\#L019571). A pool of 4 nontargeting siRNAs (ON-TARGETplus nontargeting pool, \#D001810) was used as a control. Oligo sequences are given in supplemental Table 2. Depletion of STXBP1 was assessed by immunoblot. Band intensities of immunoblots were analyzed using ImageJ (http://rsbweb.nih.gov/ij/) to determine STXBP1 knockdown efficiency.

Lentiviral transduction of endothelial cells. Lentivirus encoding monomeric enhanced green fluorescent protein (mEGFP) (LVX-mEGFPLIC) or mEGFP-STXBP1 (LVX-mEGFP-STXBP1) was produced in HEK293T cells grown in a collagen matrix. LVX-mEGFP-LIC or LVXmEGFP-STXBP1 together with VSV-G, REV, and MDL helper plasmids ${ }^{34}$ were transfected using TransIT-LT1 transfection reagent (Mirus Bio LCC, Madison, WI) essentially according to the manufacturer's recommendation. After overnight incubation, the medium was exchanged for EGM-18. Endothelial cells were transduced by 2 subsequent overnight incubations with $0.45-\mu \mathrm{m}$ filtered EGM-18 culture supernatants of virus-producing HEK293T cells.

\section{Immunoprecipitation}

HUVECs were lysed in NP40 lysis buffer (1\% NP40, 10\% glycerol, $137 \mathrm{mM}$ $\mathrm{NaCl}, 25 \mathrm{mM} N$-2-hydroxyethylpiperazine- $N^{\prime}$-2-ethanesulfonic acid, $\mathrm{pH} 7.4$, and protease/phosphate inhibitors), supplemented with $5 \mathrm{mM} \mathrm{MgCl}_{2}$ and $5 \mathrm{mM} \mathrm{CaCl}_{2}$ (STXBP1 and syntaxin-3 immunoprecipitations) or $10 \mathrm{mM}$ EDTA (GFP immunoprecipitation). Total cell lysates were centrifuged for 10 minutes at $4^{\circ} \mathrm{C}$ at $20000 \mathrm{~g}$. A total of $50 \mu \mathrm{L}$ magnetic Protein G Dynabeads was coupled with $10 \mu \mathrm{g}$ mouse anti-STXBP1 immunoglobulin G (IgG)/rabbit anti-syntaxin-3 IgG/sheep anti-GFP IgG or control mouse/rabbit/sheep IgG (Santa Cruz Biotechnology, Santa Cruz, CA) and were subsequently crosslinked using BS3 according to the manufacturer's instructions. Supernatants were incubated with antibody-coupled beads for 2 hours at $4{ }^{\circ} \mathrm{C}$ and were washed 4 times with 1\% NP40 lysis buffer. Coimmunoprecipitates and lysates were separated on precast $4 \%$ to $12 \%$ NuPAGE gels (Invitrogen) and assessed by immunoblot with primary antibodies (supplemental Table 1), followed by the appropriate infrared (IR) dye-coupled donkey secondary antibodies (LI-COR Biosciences, Cambridge, United Kingdom). Membranes were scanned with the LI-COR Odyssey Infrared Imaging system.

\section{Fluorescence microscopy}

Immunostaining and fluorescence imaging of fixed cells was performed as previously described. ${ }^{5}$ Immunostained cells were mounted in MOWIOL or Fluorsave mounting medium (Calbiochem, Nottingham, United Kingdom) and images were acquired by confocal microscopy using a Zeiss LSM 510 (Carl Zeiss, Jena, Germany) or a Leica SP1 (Leica Microsystems, Wetzlar, Germany).

Ionomycin-induced exocytosis of WPBs containing Proregion-EGFP (which essentially is VWF propeptide with a carboxy-terminal-fused EGFP $\operatorname{tag}^{35}$ ) or EGFP-Slp4-a full length (FL) or EGFP-Slp4-a-synaptotagmin-like protein homology domain (SHD) expressing WPBs was determined as previously described. $^{29,36}$ The probability of release $\left(P_{r}\right)$ was determined as the mean percentage of degranulation of fluorescent WPBs after ionomycin stimulation.

\section{Statistical analysis}

Statistical analysis was by 1-way analysis of variance and Bonferroni posttest analysis using GraphPad Prism 5.04 (GraphPad, La Jolla, CA). Significance values are shown in the figures or figure legends. Data are shown as mean \pm standard error of the mean.

\section{Results}

$\mathrm{N}$-terminal SHD mediates WPB localization but is not sufficient to enhance WPB exocytosis

Previously, we have shown that Rab27A recruits its effector Slp4-a to the WPB, which coincides with the acquisition of secretion competence by this secretory organelle. ${ }^{29}$ WPBs contain a cocktail of 
A

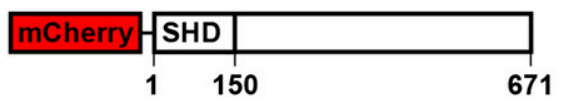

mCherry

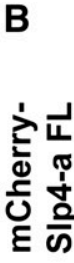

VWF propeptide
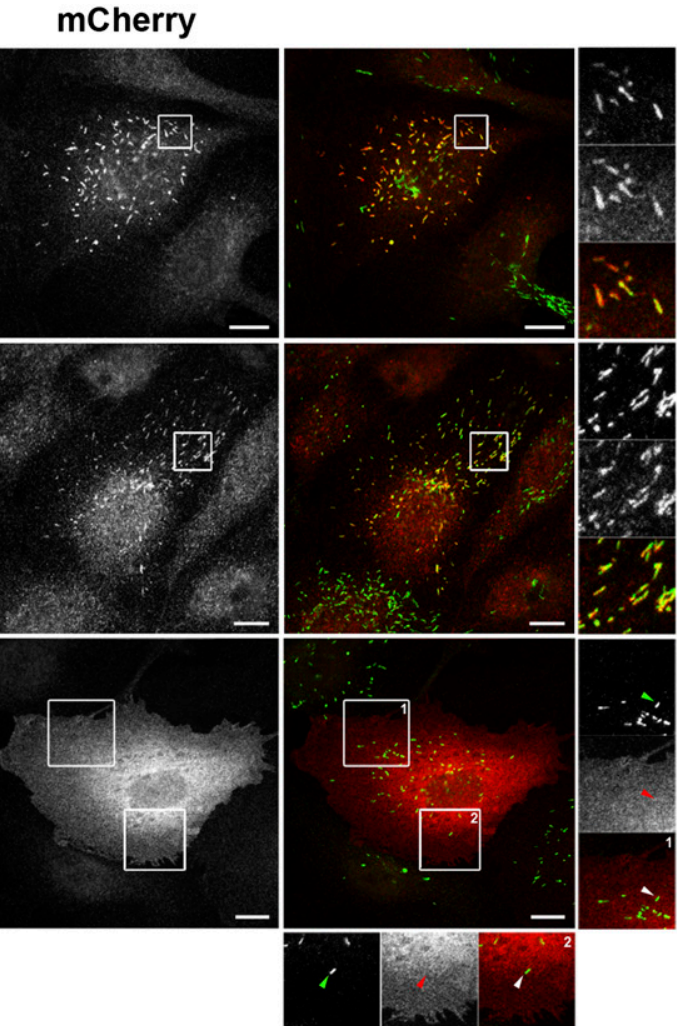

Figure 1. Targeting of SIp4-a to WPBs depends on its SIp homology domain. (A) Cartoon overview of mCherry-tagged Slp4-a truncation variants, indicating the boundaries of the SHD domain. (B) Immunocytochemical analysis of intracellular localization of SIp4-a variants. HUVECs were transfected with mCherry-tagged constructs and fixed with paraformaldehyde 24 hours posttransfection. WPBs were visualized by immunostaining for VWF propeptide (green). Magnifications of boxed regions are shown on the right and the bottom. Arrowheads point to WPBs devoid of mCherry-SIp4-a- $\Delta$ SHD. Bars represent $10 \mu \mathrm{m}$.

secretory Rabs capable of recruiting Slp4-a, including Rab27A and 3 isoforms of Rab3 ( $\operatorname{Rab} 3 \mathrm{~A}, \mathrm{~B}$, and D). ${ }^{22,23,25,29} \mathrm{Slp} 4$-a is reported to bind to these secretory Rabs via its N-terminal SHD. ${ }^{37}$ We speculated that this region was responsible for recruitment of Slp4-a to WPBs and for this purpose we expressed epitope-tagged fusion proteins of Slp4-a truncations that consist of, or lack, the SHD (Figure 1A). Full-length mCherry-Slp4-a and mCherry-Slp4-a-SHD localized to WPBs, whereas a truncation mutant lacking the SHD domain (mCherrySlp4-a- $\Delta$ SHD) did not. Instead, a diffuse cytoplasmic staining extending to the cell periphery and possibly the plasma membrane was observed (Figure 1B).

Having confirmed that the SHD domain of Slp4-a was essential for recruitment to WPBs, we next examined whether this region alone was sufficient to support the enhancement of hormone-evoked WPB exocytosis reported for EGFP-Slp4-a-FL. ${ }^{29}$ Exogenously expressed EGFP-Slp4-a-SHD labeled WPBs exclusively (Figure 2A). Data from live cell imaging of $\mathrm{Ca}^{2+}$-driven WPB exocytosis in EGFPSlp4-a-SHD-expressing HUVEC (Figure 2B-D) was compared with previously reported data for Proregion-EGFP (which labels the WPB contents and serves as a control) and EGFP-Slp4-a FL-expressing HUVECs. ${ }^{29}$ As for EGFP-Slp4-a FL, expression of EGFP-Slp4-aSHD significantly increased the delay in the onset of WPB fusion (Figure 2Di) and reduced the mean maximal rate of WPB exocytosis, although like EGFP-Slp4-a FL, this difference did not reach statistical significance (Figure 2Dii). However, in contrast to EGFP-Slp4-a FL, EGFP-Slp4-a-SHD failed to increase the $P_{r}$ of WPBs (Figure 2Diii). This suggested that the capacity to enhance $\mathrm{Ca}^{2+}$-evoked WPB exocytosis must reside in the region of the molecule distal to the SHD.

\section{STXBP1 and syntaxin-2 and -3 are targets for SIp4-a in endothelial cells}

To identify effectors of Slp4-a involved in regulated WPB release, we performed pull-downs in HUVEC lysates with glutathione S-transferase (GST)-Slp4-a- $\Delta$ SHD and GST-Slp4-a-SHD. Putative interacting proteins were identified by mass spectrometry after their separation by sodium dodecyl sulfate polyacrylamide gel electrophoresis (SDS-PAGE) and trypsin-mediated in-gel digestion (see supplemental data). As expected, several peptides derived from Slp4-a and GST were identified (Figure 3). Using the criteria outlined in the supplemental data, 324 candidate binding partners for Slp4-a$\Delta$ SHD and 429 potential interactors for Slp4-a-SHD were identified (for a full list, see supplemental Table 3A-B).

The SHD contains the WPB targeting signal for Slp4-a; therefore, we expect proteins responsible for recruitment of Slp4-a among the candidates. Partners for the SHD were Rab8A and Rab27A (Figure 3A), 2 Rab GTPases that have previously been described to be able to interact with the SHD of Slp4-a. ${ }^{37,38}$ Interestingly, the Rac1 exchange factor PREX-1, which we recently identified as a regulator of CAMP-mediated WPB release, was also pulled down. ${ }^{39}$ Annotated spectra of Rab8A and Rab27A are shown in supplemental Figures 1 and 2.

Because we determined that the exocytosis-promoting capacity of Slp4-a is localized carboxyl-terminal of the SHD, we hypothesized that among the interactome of Slp4-a, $\triangle$ SHD effectors of Slp4-a involved in exocytosis can be found. A prominent binding partner of Slp4-a- $\Delta$ SHD was STXBP1 (Figure 3A). Seventeen unique peptides corresponding to STXBP1 were identified; the annotated 
A
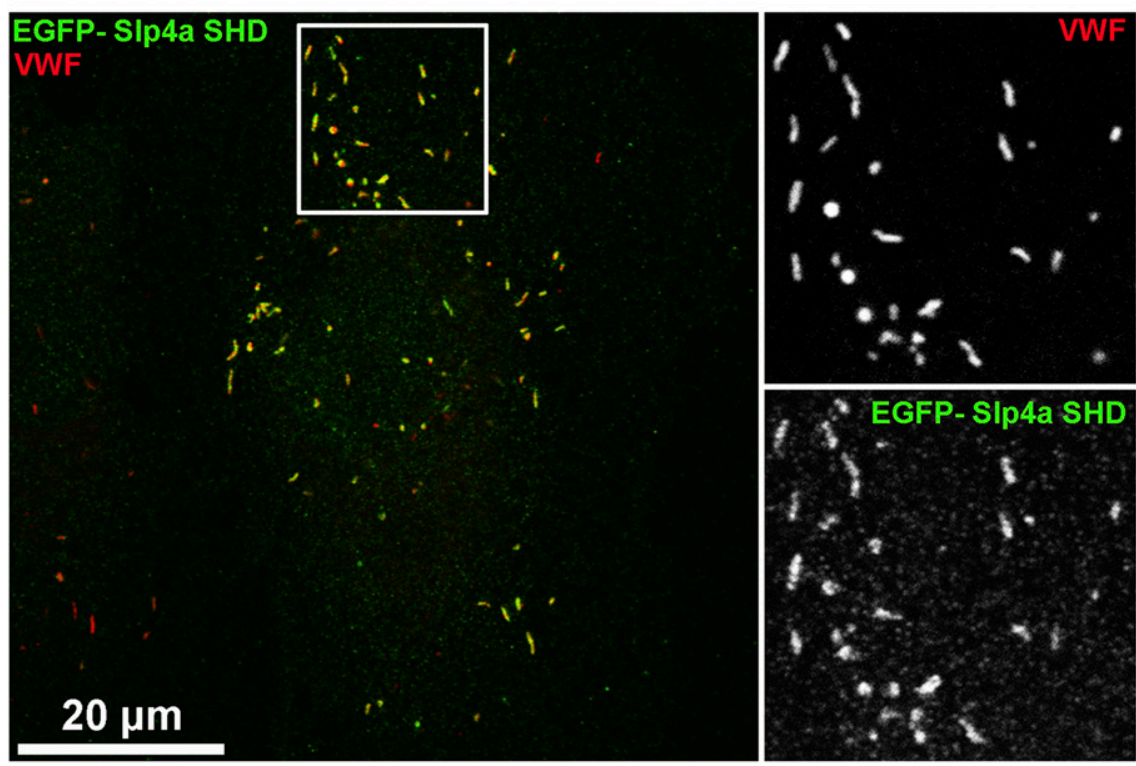

\section{B}

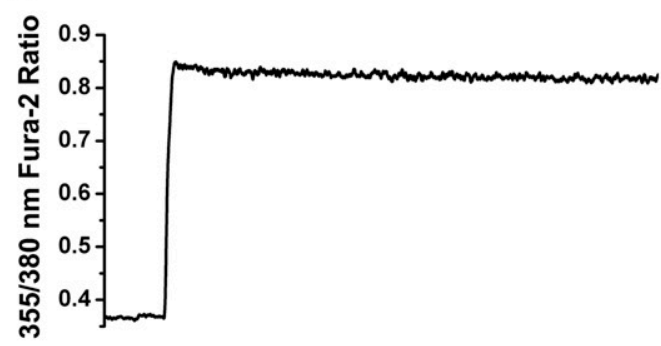

C

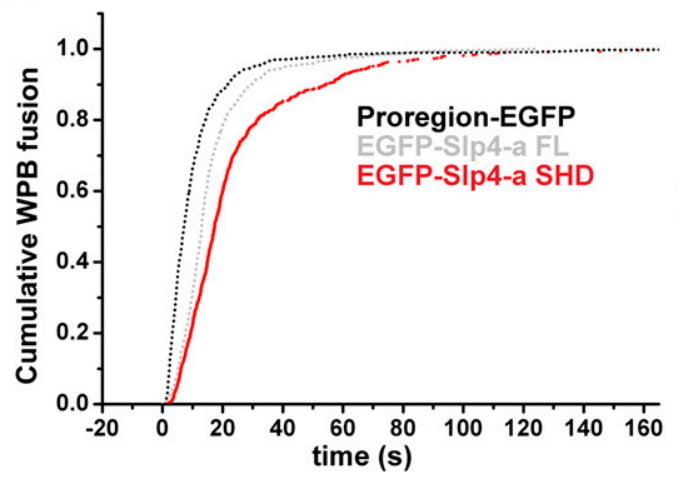

Di

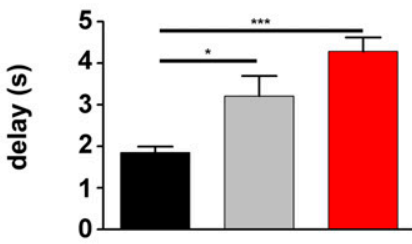

Dii

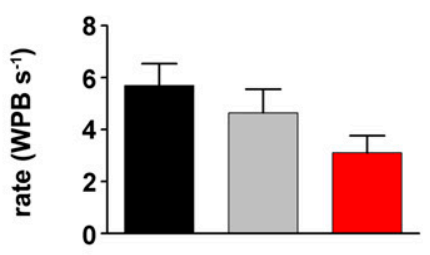

Diii

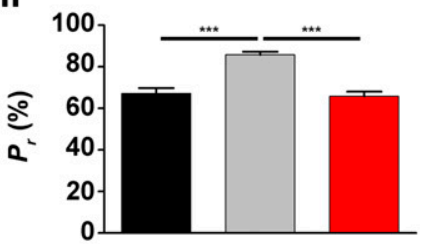

Figure 2. SIp4-a enhances WPB probability of release through a region distal to the SHD domain. (A) HUVEC expressing EGFP-SIp4-a-SHD (green) and immunolabeled for VWF (red). Grayscale images are from the region indicated by the white box. Bar represents $20 \mu \mathrm{m}$. (B) Fura-2 fluorescence ratio showing a representative record of the increase in fluorescence ratio (intracellular $\mathrm{Ca}^{2+}$ ) upon addition of $1 \mu \mathrm{M}$ ionomycin. (C) Cumulative plot of WPB fusion times, normalized by their total number, in ProregionEGFP- (black), EGFP-SIp4-a FL- (gray), and EGFPSlp4-a-SHD-expressing cells (red). (Di-iii) Mean delays (seconds), maximal rates of exocytosis (WPBs/second), and probabilities of WPB exocytosis, $P_{r},(\%)$ for ProregionEGFP- (black), EGFP-SIp4-a FL- (gray), and EGFPSlp4-a-SHD-expressing cells (red). Mean \% degranulation WPB Proregion-EGFP $=65.8 \pm 1.5 \% \mathrm{n}=46$ cells, 2151 fusion events; mean \% degranulation WPB EGFPSlp4-a $F L=85.8 \pm 2.6 \%, n=15$ cells, 849 fusion events; mean \% degranulation WPB EGFP-SIp4-a$\mathrm{SHD}=65.8 \pm 2.2 \%, \mathrm{n}=22$ cells, 694 fusion events. ${ }^{\star} P<.05,{ }^{\star \star \star} P<.005$ spectrum of peptide ISEQTYQLSR corresponding to residues 288-297 is displayed in Figure 3B. STXBP1 has previously been identified as an important regulator of secretory vesicle exocytosis because of its abilities to regulate docking of secretory granules to the plasma membrane and regulation of the SNARE complex assembly. ${ }^{40-44}$ We also identified 2 SNARE proteins: 6 unique peptides derived from syntaxin-3, and 1 peptide derived from syntaxin-2. Other highly significant candidates are Ras interacting protein 1 and Vps39. Annotated spectra of syntaxin-2 and -3 are shown in supplemental Figures 3 and 4.

Identification of STXBP1, syntaxin-2, and syntaxin-3 as putative binding partners of Slp4-a led us to test whether these proteins might exist in a complex in which the interaction of syntaxin- 2 or -3 with Slp4-a is mediated through STXBP1, as has been previously suggested. ${ }^{45}$ To confirm the interaction of STXBP1 with syntaxins and Slp4-a, we performed pull-down studies using exogenously expressed mEGFP-STXBP1 in endothelial cells. As shown in Figure 4A, STXBP1 bound endogenous Slp4-a as well as endogenous syntaxin-3. Further coimmunoprecipitation studies of endogenous STXBP1 revealed that syntaxin-2 and syntaxin-3 associate with STXBP1 in HUVECs (Figure 4B). We also assessed the ability of syntaxin-4 to interact with STXBP1, because this syntaxin has previously been implicated in the regulation of WPB exocytosis. ${ }^{46}$ However, syntaxin-4 failed to coimmunoprecipitate with STXBP1 (Figure 4B). When approached from the other end, we could also demonstrate coprecipitation of STXBP1 with syntaxin-3 (Figure 4C). Syntaxin-3 immunoprecipitation did not coprecipitate syntaxin-2, ruling out the possibility that STXBP1 binds syntaxin-2 
Figure 3. Proteomic identification of SIp4-a interactors by pull-down of GST-tagged SIp4-a truncation variants. (A) SIp4-a interactors were pulled down from HUVEC lysates by incubation with purified GSTSlp4-a-SHD (left) or GST-SIp4-a- $\Delta$ SHD (right) coupled to Glutathione-Sepharose. Eluted proteins were separated by SDS-PAGE and analyzed by mass spectrometry using the criteria described in the "Materials and methods" section. Mascot score of putative interactors (GST-SIp4-a-SHD: 429 identifications; GST-SIp4-a$\triangle$ SHD: 324 identifications) was plotted vs number of unique peptides identified. Colored symbols indicate putative interactors that have a ProHits frequency $\leq 0.0278$. Blue symbols represent bait (GST and SIp4-a), green symbols represent interactors shown in the table (bottom) that summarizes the Mascot score, number of unique peptides identified, and the ProHits frequency. (B) Annotated spectrum of the ISEQTYQLSR peptide corresponding to residues 288-297 of STXBP1.
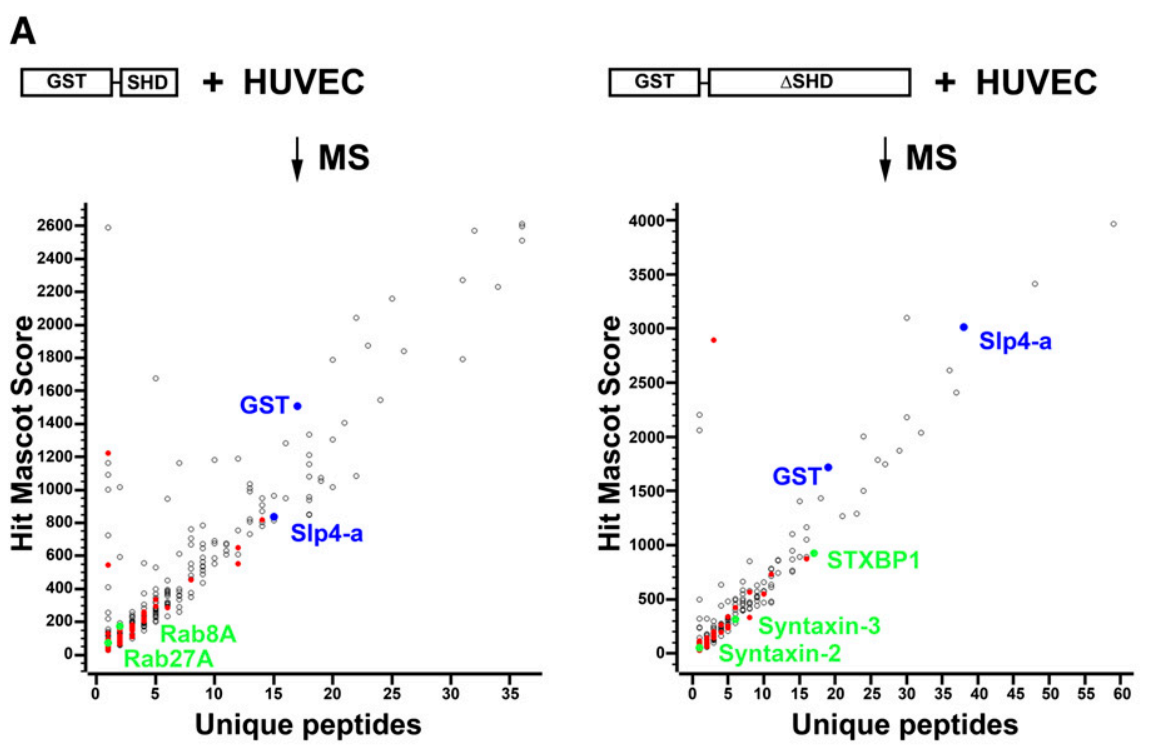

\begin{tabular}{|l|c|c|c|}
\hline Protein & Mascot & Peptides & Frequency \\
\hline GST & 1507 & 17 & 0.2037 \\
\hline Slp4-a & 836 & 15 & 0.0185 \\
\hline Rab8A & 176 & 2 & 0.0185 \\
\hline Rab27A & 74 & 1 & 0.0093 \\
\hline
\end{tabular}

\begin{tabular}{|l|c|c|c|}
\hline Protein & Mascot & Peptides & Frequency \\
\hline GST & 1718 & 19 & 0.2037 \\
\hline SIp4-a & 3014 & 38 & 0.0185 \\
\hline STXBP1 & 925 & 17 & 0.0093 \\
\hline Syntaxin-3 & 316 & 6 & 0.0093 \\
\hline Syntaxin-2 & 52 & 1 & 0.0278 \\
\hline
\end{tabular}

B
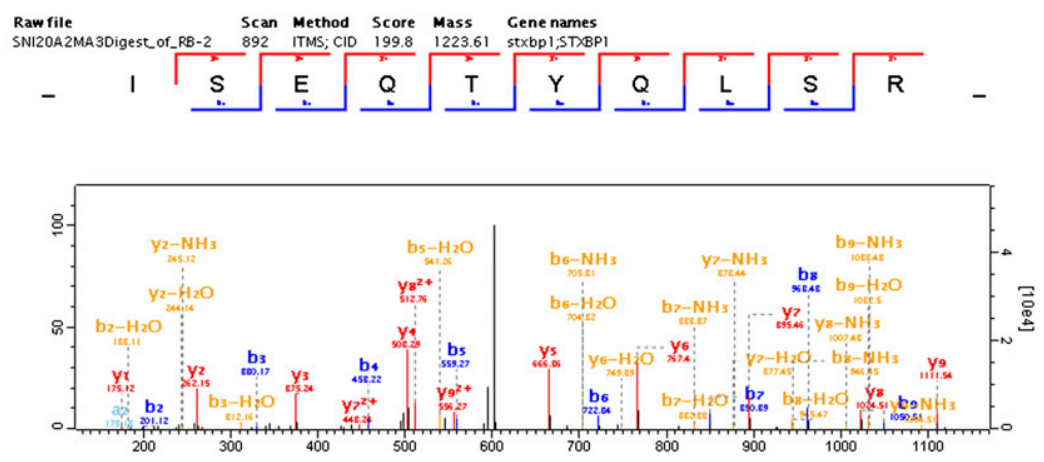

and -3 simultaneously. Taken together, the data suggest that Slp4-aSTXBP1-syntaxin-2 and Slp4-a-STXBP1-syntaxin-3 complexes are present in endothelial cells.

\section{STXBP1 depletion decreases WPB exocytosis}

To address whether STXBP1 is involved in regulating WPB exocytosis, we reduced STXBP1 in primary endothelial cells using siRNA-mediated knockdown (Figure 5A). Endogenous expression of STXBP1 protein was decreased to approximately $30 \%$ of control levels (Figure 5B). STXBP1 depletion did not affect the number or cellular localization of WPBs (not shown), nor did it affect intracellular levels of VWF (Figure 5C). Slp4-a or Rab27A localization was also not notably altered after STXBP1 knockdown (not shown). However, depletion of STXBP1 impaired both histamine- and forskolin-induced VWF secretion (Figure 5D), indicating that STXBP1 is a critical mediator for both $\mathrm{Ca}^{2+}$ - and cAMP-triggered WPB exocytosis.

\section{Impaired WPB exocytosis in BOECs derived from an EIEE4 patient}

To further test the function of STXBP1 we determined whether hormone-evoked secretion of VWF was impaired in endothelial cells derived from a patient with a known defect in STXBP1. EIEE4 is a rare and very severe epileptic disorder characterized by defective neurotransmitter release as a consequence of de novo loss-offunction mutations in the STXBP1 gene. ${ }^{30-32}$ As such, individuals with EIEE4 represent human models of STXBP1 dysfunction. We isolated BOECs from a 16-year-old EIEE4 patient carrying a de novo heterozygous 23- to 35.4-kbp microdeletion in STXBP1 [c.963+? $\left(1967+\right.$ ?)del; p.Thr322_Glu603 del]. ${ }^{30}$ This deletion affects exons 12 to 20 of STXBP1 and is predicted to result in either a truncated protein spanning amino acids 1-321 (provided that the messenger RNA transcript undergoes translation) or in nonsense mediated decay, which would result in haploinsufficiency. ${ }^{31}$ In this patient, circulating plasma levels of VWF:Ag (64 IU/dL) were at the low end of the physiological range (50-200 IU/dL). BOEC colonies were derived from peripheral blood and were pooled for further analysis. As shown in Figure 6A, endogenous STXBP1 protein expression was decreased in EIEE4 BOECs compared with BOECs derived from a healthy control individual $(49.6 \pm 5.4 \%$ residual STXBP1 protein level as estimated by immunoblotting, Figure 6B). Using 2 antibodies directed against STXBP1, we were unable to detect the predicted truncated form of STXBP1 in EIEE4 BOECs (data not shown), supporting the hypothesis that the aberrant transcript is degraded. The decrease in STXBP1 protein expression did not affect WPB morphology or numbers as shown by immunocytochemistry (Figure 6C). In addition, 
A

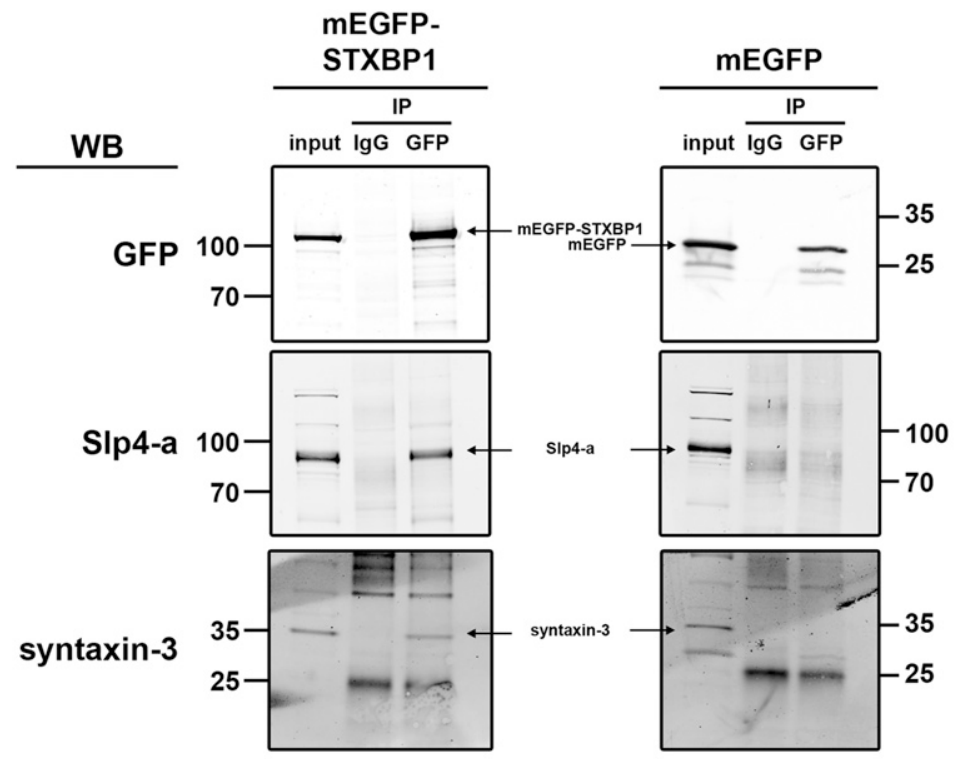

B

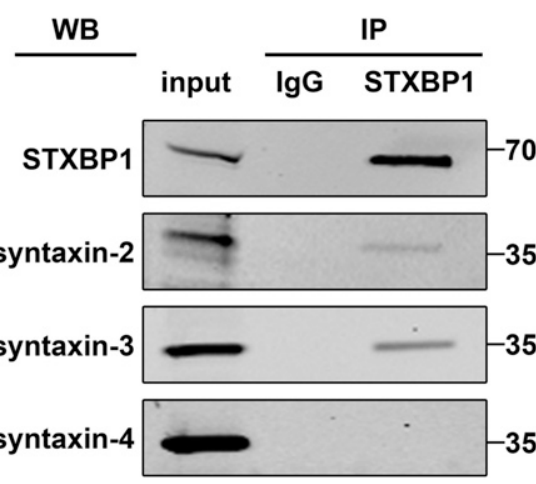

C

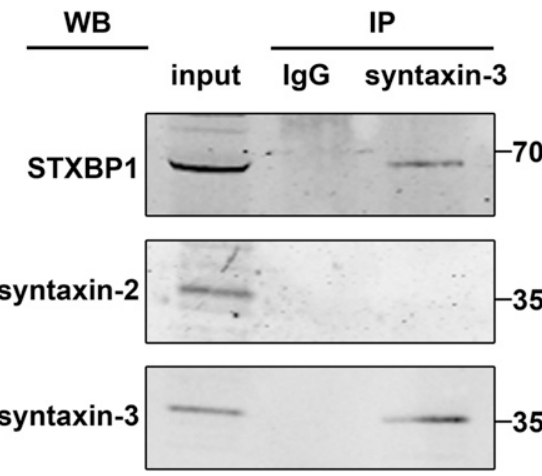

Figure 4. Interaction of STXBP1 with SNARE proteins in endothelial cells. (A) Lysates of endothelial cells lentivirally transduced with mEGFP-STXBP1 or mEGFP were incubated with magnetic beads covalently coupled with sheep anti-GFP IgG or an equivalent amount of naive sheep IgG. Lysates (input) and coimmunoprecipitates (IP) were separated by SDS-PAGE and probed with rabbit anti-SIp4-a, anti-syntaxin-3, or mouse anti-GFP followed by incubation with IR dyelabeled donkey anti-rabbit or anti-mouse IgG. SIp4 a and syntaxin- 3 bound mEGFP-STXBP1 but not mEGFP. $(B, C)$ Endothelial lysates were incubated with magnetic beads covalently coupled with mouse anti-STXBP1 IgG or an equivalent amount of naive mouse $\lg G(B)$ or with rabbit anti-syntaxin-3 IgG or an equivalent amount of naive rabbit IgG $(C)$. Lysates (input) and co- IP were separated by SDS-PAGE and probed with rabbit antiSTXBP1, anti-syntaxin-2, anti-syntaxin-3, or mouse antisyntaxin-4 followed by incubation with IR dye-labeled donkey anti-rabbit or anti-mouse IgG. Syntaxin-2 and syntaxin-3 but not syntaxin-4 coimmunoprecipitated with STXBP1 (B). Immunoprecipitation of syntaxin-3 coprecipitated STXBP1 but not syntaxin-2 (C).

we did not observe changes in the recruitment of Slp4-a or Rab27A to WPBs (supplemental Figures 5 and 6), suggesting that the upstream components of the exocytotic machinery were unperturbed. EIEE4 BOECs contained slightly more VWF when compared with healthy

A

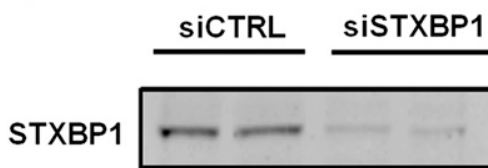

Figure 5. Impaired $\mathrm{Ca}^{2+}$ - and cAMP-mediated VWF secretion after STXBP1 depletion. (A) HUVECs were nucleofected with siRNA oligos directed against STXBP1 (siSTXBP1) or with nonhybridizing control oligos (siCTRL) and were assayed 48 hours after nucleofection. Lysates were separated by SDS-PAGE and were probed with rabbit anti-STXBP1 or mouse anti-actin antibodies followed by incubation with IR dye-labeled donkey anti-rabbit or anti-mouse IgG. (B) Quantification of STXBP1 expression in control cells (black) or after STXBP1 depletion (white) $(n=3)$. (C) Endothelial cells were lysed in SF medium containing $1 \%$ Triton $X-100$ and were assayed for VWF content by ELISA $(n=3)$. (D) Endothelial cells were incubated for 30 minutes with SF medium (basal), SF medium supplemented with $10 \mu \mathrm{M}$ forskolin, and $100 \mu \mathrm{M}$ IBMX (FSK) or with $100 \mu \mathrm{M}$ histamine (HIS). Supernatants were assayed for secreted VWF by ELISA. ${ }^{\star \star \star} P<.005$ $(n=3)$. donor BOECs; however, this difference was not statistically significant (Figure 6D). We next tested whether agonist-induced release of VWF was impaired in EIEE4 BOECs. Unstimulated (basal) VWF secretion was slightly lower in EIEE4 BOECs, but this was not significant 
Figure 6. Impaired $\mathrm{Ca}^{2+}$ - and cAMP-mediated VWF secretion in EIEE4 BOECs. (A) Healthy control donor and EIEE4 BOEC lysates were separated by SDSPAGE and were probed with rabbit anti-STXBP1 or mouse anti-actin antibodies followed by incubation with IR dye-labeled donkey anti-rabbit or anti-mouse IgG. (B) Quantification of STXBP1 expression in healthy control donor (black) or EIEE4 BOECs (white) $(n=3)$. (C) EIEE4 and control donor BOECs were fixed with paraformaldehyde and immunostained for VWF (green) and VE-cadherin (red). Bar represents $10 \mu \mathrm{m}$. (D) Endothelial cells were lysed in SF medium containing $1 \%$ Triton X-100 and lysates of healthy control donor (black) or EIEE4 BOECs (white) were assayed for VWF content by ELISA. (E) BOECs were incubated for 30 minutes with SF medium (basal), SF medium supplemented with $10 \mu \mathrm{M}$ forskolin and $100 \mu \mathrm{M}$ IBMX (FSK), or with $100 \mu \mathrm{M}$ histamine (HIS). Supernatants were assayed for secreted VWF by ELISA. ${ }^{* \star \star} P<.005 ; \mathrm{n}=3$.
A

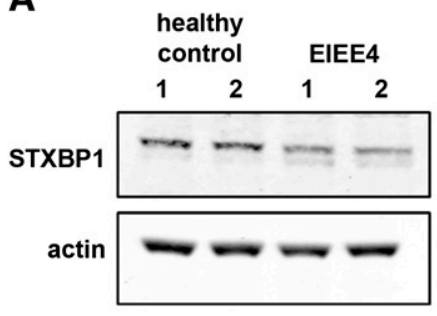

B

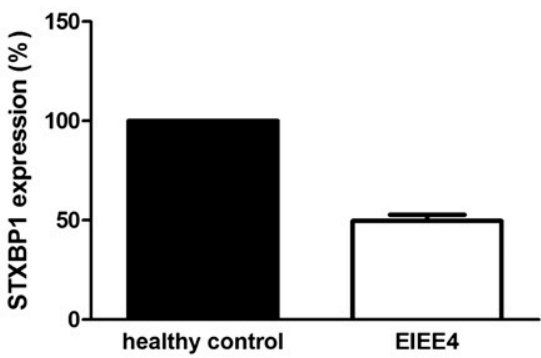

C

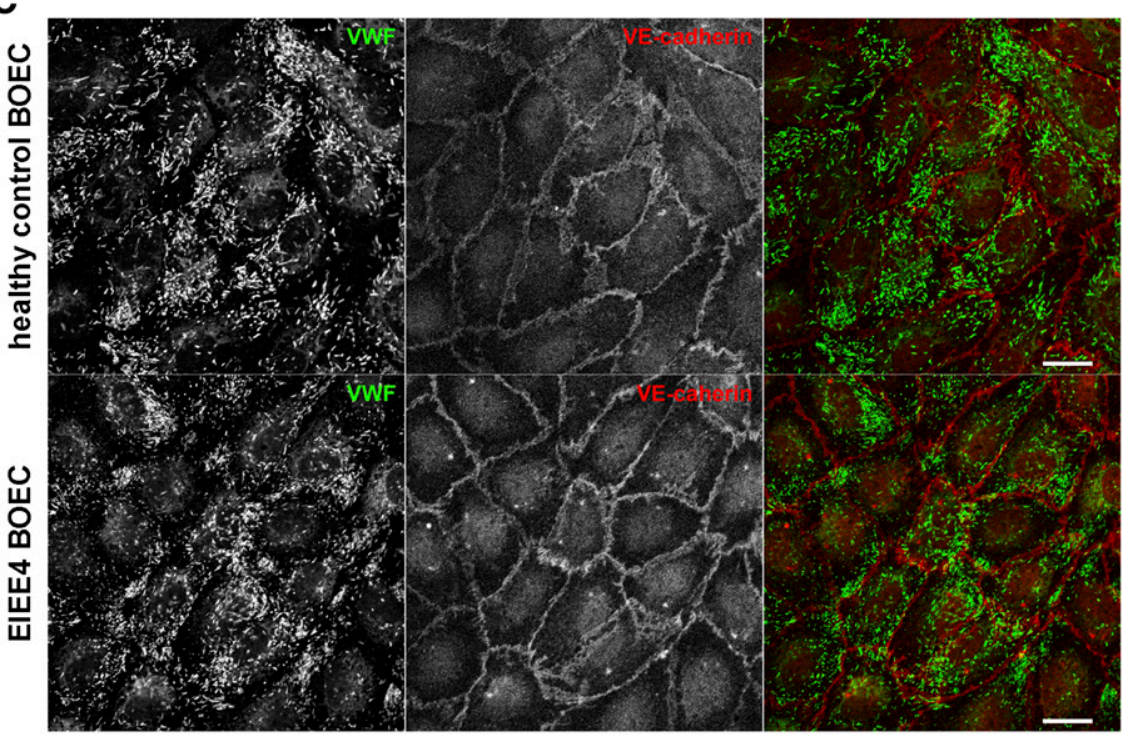

D

E

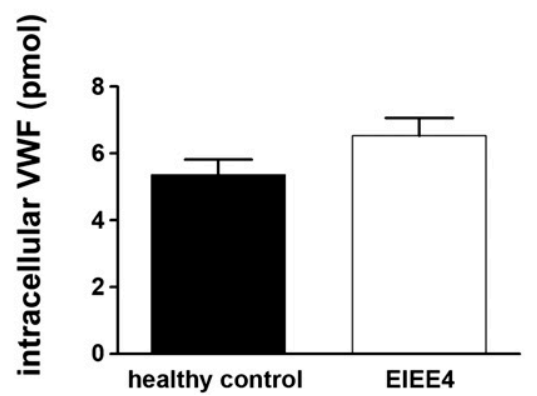

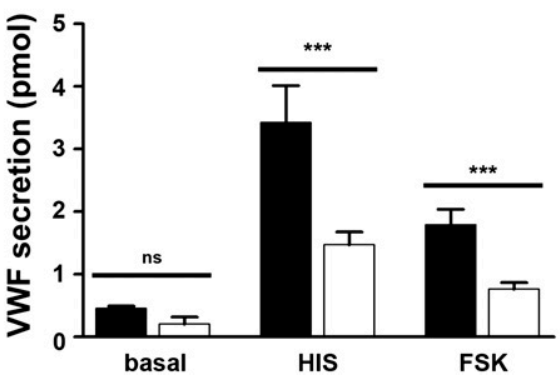

compared with the control BOECs of a healthy donor. However, both histamine- and forskolin-stimulated VWF secretion were significantly reduced in the EIEE4 BOECs compared with the control (Figure 6E).

\section{Discussion}

The small GTPase Rab27A and its effectors MyRIP, Munc13-4, and Slp4-a play key roles as regulators of WPB exocytosis. ${ }^{25,27-29}$ Each of these effectors perform discrete steps in the sequence of events that follow secretory organelle formation until eventual exocytosis, with some of them exerting opposing effects (MyRIP-Myosin Va vs Slp4-a and Munc13-4) on the probability of release of the WPB. A complicated picture emerges, in which the releasability of a WPB is determined by the balance of effectors present on its membrane ${ }^{29}$ and the efficacy of the individual effectors' downstream mechanisms. Although the mechanism of action of MyRIP, a negative-regulator of
WPB exocytosis, has been partially elucidated, ${ }^{28}$ how Slp4-a and Munc13-4 function as positive regulators of WPB exocytosis remains unclear. Here we provide evidence that Slp4-a functions through the recruitment of STXBP1.

The SHD-domain of Slp4-a contains binding sites for several secretory Rabs, including Rab27A, Rab3, and Rab8. Consistent with this, our unbiased proteomic analysis identified Rab27A and Rab8A as binding partners for the SHD domain of Slp4-a. Earlier work from our group has shown that WPB localization of Slp4-a depends on the small GTPases Rab27A and (primarily) Rab3B; however, only Rab27A-bound Slp4-a was found to be functionally involved in exocytosis. Surprisingly, we did not identify peptides of any of the Rab3 isoforms in our pull-down with Slp4-a-SHD, although fluorescence recovery after photobleaching studies suggest that the majority of WPB localized Slp4-a is in complex with Rab3B. ${ }^{29}$ The likely explanation is that the Slp4-a-Rab3 interaction is weaker or more labile than the corresponding interactions with Rab27A or Rab8. This idea is supported by both coimmunoprecipitation studies 


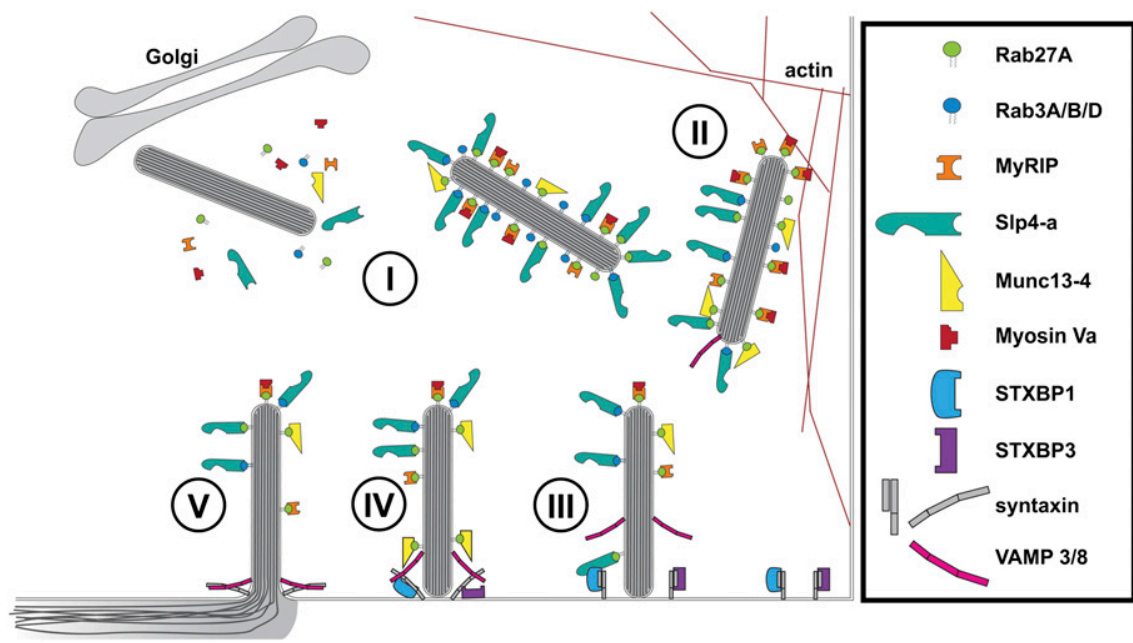

Figure 7. Cartoon model of regulation of WPB exocytosis. (I) Newly formed WPBs recruit Rab proteins (Rab27A and Rab3A/B/D) and their effectors SIp4-a, Munc13-4, and MyRIP/Myosin Va. ${ }^{22,23,25,27-29}$ (II) WPBs are anchored to the actin cytoskeleton via Myosin Va and MyRIP. ${ }^{27,28}$ (III) STXBP1 links WPBs to the SNAREs (syntaxin-2/3) via Slp4-a and Rab27A, whereas STXBP3 (Munc18c) associates with syntaxin-4. ${ }^{46}$ (IV) SNARE complex assembly of syntaxins with WPBassociated VAMP $3 / 8,{ }^{59}$ potentially facilitated by Munc13$4 .^{25}$ Fusion of WPB and plasma membrane and dispersal of WPB cargo into vascular lumen.

that show a very weak pull-down of Rab3s compared with Rab27A and by fluorescence recovery after photobleaching analysis of WPB dissociation rates for Slp4-a in the presence of exogenously expressed Rab27A, Rab3B, or Rab3D. ${ }^{29}$ In the latter case, the dissociation rate for Slp4-a in the presence of Rab27A was an order or magnitude slower than in the presence of Rab3B or Rab3D, indicating a strong interaction between these molecules in living cells. Similar observations have been made in PC12 cells; although Rab3A was shown to interact with Slp4-a in an in vitro binding assay in the absence of Rab27A and Rab8A, ${ }^{38}$ only Rab27A and Rab8A could be detected after coimmunoprecipitation with Slp4-a. ${ }^{47}$ The strong interaction between Rab27A and Slp4-a might reflect in part the unique ability of this Rab protein to bind Slp4-a irrespective of its GTP/guanosine diphosphate status. ${ }^{37}$ Together, these properties may have contributed to a competitive advantage for Rab27A during our pull-down procedure. We also identified Rab8A in our screen, a protein that is localized to the Golgi apparatus and is not recruited to WPBs, which makes it an unlikely candidate to contribute directly to WPB localization of Slp4-a. ${ }^{48}$ Rab8A-bound Slp4-a has been reported to exert different effects on secretion depending on the cell type studied. ${ }^{47,49,50}$ Nonetheless, depletion of Rab8A has been found to decrease phorbol ester-induced VWF release from endothelial cells ${ }^{48}$; whether this is mediated via Slp4-a remains to be established.

Live cell imaging of WPB exocytosis in EGFP-Slp4-a-SHDexpressing cells showed that the SHD domain of Slp4-a alone was not sufficient to enhance hormone-evoked WPB exocytosis. This result suggests 2 items: (1) that the capacity of exogenously expressed Slp4-a to promote WPB exocytosis is unlikely to be due solely to displacement of the negative regulator MyRIP from the WPB, ${ }^{29}$ but must instead reflect an active property of Slp4-a; and (2) that this activity requires the region of the molecule distal to the SHD. We identified STXBP1, syntaxin-2, and syntaxin-3 as endogenous molecules that interact specifically with this distal region of Slp4-a. STXBPs and syntaxins are key components of the SNARE machinery, a multiprotein complex that catalyzes the fusion of membranes. ${ }^{51}$

Our data suggest a crucial role for STXBP1 in both $\mathrm{Ca}^{2+}$ - and cAMP-mediated VWF secretion from endothelial cells. In addition, stimulated VWF secretion of BOECs derived from an EIEE4 patient, a human model of STXBP1 haploinsufficiency, was impaired. Previous studies demonstrate that STXBP1 is essential for secretory vesicle release, illustrated by the fact that $S T X B P 1$-null mutant mice showed reduction of $\mathrm{Ca}^{2+}$-regulated synaptic vesicle exocytosis from neuroendocrine cells. ${ }^{42,43}$ Furthermore, insulin granule docking and secretion from pancreatic $\beta$ cells as well as catecholamine-containing large, dense, core vesicle docking and secretion from chromaffin cells are impaired by STXBP1 deletion. ${ }^{40,41,44}$ The severe neurological defects displayed by patients with EIEE4 are also attributed to abnormalities in synaptic vesicle release. ${ }^{30,31}$ On this basis, it is likely that STXBP1 also contributes to the process of WPB docking in endothelial cells, most likely by linking the WPB (through Rab27ASlp4-a) to plasma membrane syntaxins.

The physiological significance of regulation of WPB exocytosis by SNARE proteins is also underscored by a number of recent genomewide association studies on VWF plasma levels and associated risk factors for cardiovascular diseases such as arterial and venous thrombosis. Single nucleotide polymorphisms in syntaxin-2 and also STXBP5 (a member of the same protein family as STXBP1) have been associated with circulating VWF levels, risk of venous thrombosis, and severity of the bleeding phenotype in von Willebrand disease type 1 patients. ${ }^{52-55}$ It is intriguing to speculate that these deficits reflect disruption of the SNARE machinery regulating WPB exocytosis and VWF secretion.

In the EIEE4 patient studied, plasma VWF levels were found to be on the low side of the normal range. Such a mild quantitative deficiency in VWF does not qualify as von Willebrand disease type 1 according to current laboratory criteria, ${ }^{56}$ nor does it confer a high risk of bleeding; indeed, this patient has no such history. Patients with EIEE4 display severe neurological defects including early-onset epilepsy and intellectual disability, which have been attributed to abnormalities in synaptic vesicle release. ${ }^{30,31}$ STXBP1 has also been implicated in insulin release. ${ }^{40}$ Although moderately elevated levels of blood glucose have been observed in mice heterozygous for STXBP1 deficiency as a result of slightly decreased insulin release, ${ }^{40}$ no altered glucose levels or symptoms related to aberrant insulin secretion have so far been reported in patients with EIEE4. Circulating levels of VWF are determined primarily by unstimulated (basal) secretion of VWF from endothelial cells, which is now known to arise primarily through basal WPB exocytosis. ${ }^{57}$ It is interesting to speculate whether the small (but nonsignificant) reduction in basal secretion of VWF identified in cultured BOECs isolated from the EIEE4 patient might reflect the function of the patient's own endothelium, accounting for a slightly low circulating level of plasma VWF. However, we need to take into consideration that it is difficult to draw firm conclusions solely based on BOEC data of a limited number of donors because significant heterogeneity seems to exist between single isolates. ${ }^{58}$ Furthermore, based on our findings with EIEE4 BOECs, we hypothesize that the 
effect of STXBP1 haploinsufficiency on VWF secretion would present itself more prominently upon acute endothelial stimulation (eg, after administration with DDAVP).

A growing number of molecules have been identified as components of the control mechanism for stimulated WPB exocytosis (Figure 7). Earlier work from $\mathrm{Fu}$ and colleagues has shown the involvement of Munc18c (STXBP3) and syntaxin-4 in thrombinstimulated $\mathrm{P}$-selectin expression, which coincides with release of a Munc18c-syntaxin-4 interaction after protein kinase $\mathrm{C}$-dependent phosphorylation events on either partner. ${ }^{46}$ Syntaxin-4 associates with SNAP23 and the R-SNARE VAMP3 that, along with VAMP8 (which does not associate with syntaxin-4), is localized on WPBs. ${ }^{59}$ From these observations and ours, it becomes apparent that there is both redundancy within the SNARE molecules involved as well as a considerable resilience of endothelial cells to conditions that perturb SNARE complex formation. ${ }^{59,60}$ The apparent redundancy of SNARE complexes operating in endothelial cells might allow for different upstream signaling cascades to target discrete exocytotic machineries, providing the endothelium with the possibility to fine tune its secretory response.

\section{Acknowledgments}

The authors thank Professor Matthijs Verhage (Vrije Universiteit University Amsterdam, The Netherlands) who provided pM181b-IRES2-EGFP, Melanie Scarisbrick for assistance with purification of GST-Slp4-a truncations, Dr Arvid Suls for helpful discussions regarding the EIEE4 STXBP1 mutation, and the EIEE4 patient and family for their cooperation and donation of material for this study.

This work was supported by grants from The Netherlands Organization for Scientific Research (NWO-TOP 91209006), the Landsteiner Stichting voor Bloedtransfusie Research (LSBR-1244), the United Kingdom Medical Research Council (grants 117570589 and U117573808) (M.J.H., T.C.), and the European Hematology Association Research Fellowship (R.B.).

\section{Authorship}

Contribution: D.v.B., A.P.S., N.H., K.W.E.M.v.H., and R.B. performed research and analyzed data; S.W., B.C., P.D.J., J.E., and K.V. contributed vital reagents; D.v.B., N.H., M.F.-B., J.V., M.H., T.C., and R.B. designed the research; and D.v.B., A.P.S., J.V., T.C., and R.B. wrote the paper.

Conflict-of-interest disclosure: The authors declare no competing financial interests.

Correspondence: Ruben Bierings, Department of Plasma Proteins, Sanquin-AMC Landsteiner Laboratory, Plesmanlaan 125, 1066CX Amsterdam, The Netherlands; e-mail: r.bierings@sanquin.nl; and Tom Carter, Molecular Cell Sciences Research Centre, St. George's, University of London, Cranmer Terrace, London SW17 ORE, United Kingdom; e-mail: tcarter@sgul.ac.uk.

\section{References}

1. Lowenstein CJ, Morrell CN, Yamakuchi M. Regulation of Weibel-Palade body exocytosis Trends Cardiovasc Med. 2005;15(8):302-308.

2. Michaux G, Cutler DF. How to roll an endothelial cigar: the biogenesis of Weibel-Palade bodies. Traffic. 2004;5(2):69-78.

3. Rondaij MG, Bierings R, Kragt A, van Mourik JA, Voorberg J. Dynamics and plasticity of WeibelPalade bodies in endothelial cells. Arterioscler Thromb Vasc Biol. 2006;26(5):1002-1007.

4. Bonfanti R, Furie BC, Furie B, Wagner DD. PADGEM (GMP140) is a component of WeibelPalade bodies of human endothelial cells. Blood. 1989;73(5):1109-1112.

5. Knipe L, Meli A, Hewlett L, et al. A revised model for the secretion of tPA and cytokines from cultured endothelial cells. Blood. 2010;116(12): 2183-2191.

6. McEver RP, Beckstead JH, Moore KL, Marshall-Carlson L, Bainton DF. GMP-140, a platelet alpha-granule membrane protein, is also synthesized by vascular endothelial cells and is localized in Weibel-Palade bodies. J Clin Invest. 1989;84(1):92-99.

7. Utgaard JO, Jahnsen FL, Bakka A, Brandtzaeg P, Haraldsen G. Rapid secretion of prestored interleukin 8 from Weibel-Palade bodies of microvascular endothelial cells. J Exp Med. 1998; 188(9):1751-1756

8. Vischer UM, Wagner DD. CD63 is a component of Weibel-Palade bodies of human endothelial cells. Blood. 1993;82(4):1184-1191.

9. Wolff B, Burns AR, Middleton J, Rot A. Endothelial cell "memory" of inflammatory stimulation: human venular endothelial cells store interleukin 8 in Weibel-Palade bodies. J Exp Med. 1998;188(9): 1757-1762.

10. Doyle EL, Ridger V, Ferraro F, Turmaine M, Saftig $P$, Cutler DF. CD63 is an essential cofactor to leukocyte recruitment by endothelial P-selectin Blood. 2011;118(15):4265-4273.

11. Fiedler $U$, Scharpfenecker $M$, Koidl S, et al. The Tie-2 ligand angiopoietin-2 is stored in and rapidly released upon stimulation from endothelial cell Weibel-Palade bodies. Blood. 2004;103(11): 4150-4156

12. Starke RD, Ferraro F, Paschalaki KE, et al. Endothelial von Willebrand factor regulates angiogenesis. Blood. 2011;117(3):1071-1080.

13. van Breevoort $D$, van Agtmaal EL, Dragt $B S$, et al. Proteomic screen identifies IGFBP7 as a novel component of endothelial cell-specific WeibelPalade bodies. J Proteome Res. 2012;11(5): 2925-2936.

14. Bierings $R$, van den Biggelaar $M$, Kragt $A$, Mertens K, Voorberg J, van Mourik JA. Efficiency of von Willebrand factor-mediated targeting of interleukin-8 into Weibel-Palade bodies. J Thromb Haemost. 2007;5(12):2512-2519.

15. Øynebråten I, Bakke $O$, Brandtzaeg $P$, Johansen FE, Haraldsen G. Rapid chemokine secretion from endothelial cells originates from 2 distinct compartments. Blood. 2004;104(2):314-320.

16. van Agtmaal EL, Bierings $R$, Dragt $B S$, et al. The shear stress-induced transcription factor KLF2 affects dynamics and angiopoietin-2 content of Weibel-Palade bodies. PLOS ONE. 2012;7(6): e38399.

17. Kaufmann JE, Oksche A, Wollheim CB, Günther G, Rosenthal W, Vischer UM. Vasopressininduced von Willebrand factor secretion from endothelial cells involves V2 receptors and cAMP. $J$ Clin Invest. 2000;106(1):107-116.

18. Loesberg C, Gonsalves MD, Zandbergen J, et al. The effect of calcium on the secretion of factor VIIIrelated antigen by cultured human endothelial cells. Biochim Biophys Acta. 1983;763(2):160-168.

19. van Hooren KW, van Agtmaal EL, FernandezBorja M, van Mourik JA, Voorberg J, Bierings R.
The Epac-Rap1 signaling pathway controls cAMP-mediated exocytosis of Weibel-Palade bodies in endothelial cells. $J$ Biol Chem. 2012; 287(29):24713-24720.

20. Vischer UM, Wollheim CB. Epinephrine induces von Willebrand factor release from cultured endothelial cells: involvement of cyclic AMP dependent signalling in exocytosis. Thromb Haemost. 1997;77(6):1182-1188.

21. de Leeuw HP, Fernandez-Borja M, Reits EA, et al Small GTP-binding protein Ral modulates regulated exocytosis of von Willebrand factor by endothelial cells. Arterioscler Thromb Vasc Biol. 2001;21(6):899-904.

22. Hannah MJ, Hume AN, Arribas M, et al. WeibelPalade bodies recruit Rab27 by a content-driven, maturation-dependent mechanism that is independent of cell type. J Cell Sci. 2003; 116(Pt 19):3939-3948.

23. Knop M, Aareskjold E, Bode G, Gerke V. Rab3D and annexin A2 play a role in regulated secretion of $\mathrm{VWF}$, but not tPA, from endothelial cells. $E M B O$ J. 2004;23(15):2982-2992.

24. Rondaij MG, Bierings R, van Agtmaal EL, et al. Guanine exchange factor RalGDS mediates exocytosis of Weibel-Palade bodies from endothelial cells. Blood. 2008;112(1):56-63.

25. Zografou S, Basagiannis D, Papafotika A, et al. A complete Rab screening reveals novel insights in Weibel-Palade body exocytosis. J Cell Sci. 2012;125(Pt 20):4780-4790.

26. Fukuda M. Rab27 effectors, pleiotropic regulators in secretory pathways. Traffic. 2013;14(9): 949-963.

27. Nightingale TD, Pattni K, Hume AN, Seabra MC Cutler DF. Rab27a and MyRIP regulate the amount and multimeric state of VWF released from endothelial cells. Blood. 2009;113(20): 5010-5018. 
28. Rojo Pulido I, Nightingale TD, Darchen F, Seabra MC, Cutler DF, Gerke V. Myosin Va acts in concert with Rab27a and MyRIP to regulate acute von-Willebrand factor release from endothelial cells. Traffic. 2011;12(10):1371-1382.

29. Bierings $\mathrm{R}$, Hellen $\mathrm{N}$, Kiskin $\mathrm{N}$, et al. The interplay between the Rab27A effectors SIp4-a and MyRIP controls hormone-evoked Weibel-Palade body exocytosis. Blood. 2012;120(13):2757-2767.

30. Deprez L, Weckhuysen S, Holmgren P, et al. Clinical spectrum of early-onset epileptic encephalopathies associated with STXBP1 mutations. Neurology. 2010;75(13):1159-1165.

31. Saitsu $H$, Kato $M$, Mizuguchi $T$, et al. De novo mutations in the gene encoding STXBP1 (MUNC18-1) cause early infantile epileptic encephalopathy. Nat Genet. 2008;40(6):782-788.

32. Weckhuysen $S$, Holmgren $P$, Hendrickx R, et al. Reduction of seizure frequency after epilepsy surgery in a patient with STXBP1 encephalopathy and clinical description of six novel mutation carriers. Epilepsia. 2013;54(5):e74-e80.

33. Martin-Ramirez J, Hofman M, van den Biggelaar M, Hebbel RP, Voorberg J. Establishment of outgrowth endothelial cells from peripheral blood. Nat Protoc. 2012;7(9):1709-1715.

34. Dull T, Zufferey R, Kelly M, et al. A thirdgeneration lentivirus vector with a conditional packaging system. J Virol. 1998;72(11): 8463-8471.

35. Hannah MJ, Skehel P, Erent M, Knipe L, Ogden D, Carter T. Differential kinetics of cell surface loss of von Willebrand factor and its propolypeptide after secretion from WeibelPalade bodies in living human endothelial cells. J Biol Chem. 2005;280(24):22827-22830.

36. Erent M, Meli A, Moisoi N, et al. Rate, extent and concentration dependence of histamine-evoked Weibel-Palade body exocytosis determined from individual fusion events in human endothelial cells. J Physiol. 2007;583(Pt 1):195-212.

37. Fukuda M. SIp4-a/granuphilin-a inhibits densecore vesicle exocytosis through interaction with the GDP-bound form of Rab27A in PC12 cells. J Biol Chem. 2003;278(17):15390-15396.

38. Kuroda TS, Fukuda M, Ariga $\mathrm{H}$, Mikoshiba K. The SIp homology domain of synaptotagmin-like proteins 1-4 and Slac2 functions as a nove Rab27A binding domain. J Biol Chem. 2002; 277(11):9212-9218.

39. van Hooren KW, van Breevoort D, FernandezBorja M, et al. Phosphatidylinositol-3,4,5- triphosphate-dependent Rac exchange factor 1 (PREX1) regulates epinephrine induced exocytosis of Weibel-Palade bodies. J Thromb Haemost. 2014;12(2):273-281.

40. Oh E, Kalwat MA, Kim MJ, Verhage M, Thurmond DC. Munc18-1 regulates first-phase insulin release by promoting granule docking to multiple syntaxin isoforms. J Biol Chem. 2012;287(31): 25821-25833.

41. Tomas A, Meda P, Regazzi R, Pessin JE, Halban PA. Munc 18-1 and granuphilin collaborate during insulin granule exocytosis. Traffic. 2008;9(5): 813-832.

42. Toonen RF, Wierda K, Sons MS, et al Munc18-1 expression levels control synapse recovery by regulating readily releasable pool size. Proc Natl Acad Sci USA. 2006;103(48) 18332-18337.

43. Verhage M, Maia AS, Plomp JJ, et al. Synaptic assembly of the brain in the absence of neurotransmitter secretion. Science. 2000 287(5454):864-869.

44. Voets T, Toonen RF, Brian EC, et al. Munc18-1 promotes large dense-core vesicle docking. Neuron. 2001;31(4):581-591.

45. Fukuda $M$, Imai $A$, Nashida $T$, Shimomura $H$ SIp4-a/granuphilin-a interacts with syntaxin-2/3 in a Munc18-2-dependent manner. $J$ Biol Chem. 2005;280(47):39175-39184.

46. Fu J, Naren AP, Gao X, Ahmmed GU, Malik AB Protease-activated receptor-1 activation of endothelial cells induces protein kinase Calphadependent phosphorylation of syntaxin 4 and Munc18c: role in signaling p-selectin expression. J Biol Chem. 2005;280(5):3178-3184.

47. Fukuda M, Kanno E, Saegusa C, Ogata $Y$, Kuroda TS. Slp4-a/granuphilin-a regulates densecore vesicle exocytosis in PC12 cells. J Biol Chem. 2002;277(42):39673-39678.

48. Michaux G, Dyer CE, Nightingale TD, Gallaud E, Nurrish S, Cutler DF. A role for Rab10 in von Willebrand factor release discovered by an AP-1 interactor screen in C. elegans. J Thromb Haemost. 2011;9(2):392-401.

49. Gálvez-Santisteban $M$, Rodriguez-Fraticelli $A E$, Bryant DM, et al. Synaptotagmin-like proteins control the formation of a single apical membrane domain in epithelial cells. Nat Cell Biol. 2012 14(8):838-849.

50. Hampson A, O'Connor A, Smolenski A. Synaptotagmin-like protein 4 and Rab8 interact and increase dense granule release in platelets. J Thromb Haemost. 2013;11(1):161-168.

51. Jahn R, Fasshauer D. Molecular machines governing exocytosis of synaptic vesicles. Nature. 2012;490(7419):201-207.

52. Smith NL, Chen MH, Dehghan A, et al; Wellcome Trust Case Control Consortium. Novel associations of multiple genetic loci with plasma levels of factor VII, factor VIII, and von Willebrand factor: the CHARGE (Cohorts for Heart and Aging Research in Genome Epidemiology) Consortium. Circulation. 2010;121(12):1382-1392.

53. Smith NL, Rice KM, Bovill EG, et al. Genetic variation associated with plasma von Willebrand factor levels and the risk of incident venous thrombosis. Blood. 2011;117(22):6007-6011.

54. van Loon JE, Leebeek FW, Deckers JW, et al. Effect of genetic variations in syntaxin-binding protein-5 and syntaxin-2 on von Willebrand factor concentration and cardiovascular risk. Circ Cardiovasc Genet. 2010;3(6):507-512.

55. van Loon JE, Sanders YV, de Wee EM, Kruip MJ, de Maat MP, Leebeek FW. Effect of genetic variation in STXBP5 and STX2 on von Willebrand factor and bleeding phenotype in type 1 von Willebrand disease patients. PLOS ONE. 2012; 7(7):e40624.

56. Sadler JE, Rodeghiero F; ISTH SSC Subcommittee on von Willebrand Factor. Provisional criteria for the diagnosis of VWD type 1. J Thromb Haemost. 2005;3(4):775-777.

57. Giblin JP, Hewlett LJ, Hannah MJ. Basal secretion of von Willebrand factor from human endothelial cells. Blood. 2008;112(4):957-964.

58. Wang JW, Bouwens EA, Pintao MC, et al. Analysis of the storage and secretion of von Willebrand factor in blood outgrowth endothelial cells derived from patients with von Willebrand disease. Blood. 2013;121(14): 2762-2772.

59. Pulido IR, Jahn R, Gerke V. VAMP3 is associated with endothelial Weibel-Palade bodies and participates in their $\mathrm{Ca}(2+)$-dependent exocytosis. Biochim Biophys Acta. 2011;1813(5): 1038-1044.

60. Cookson EA, Conte IL, Dempster J, Hannah MJ Carter T. Characterisation of Weibel-Palade body fusion by amperometry in endothelial cells reveals fusion pore dynamics and the effect of cholesterol on exocytosis. J Cell Sci. 2013;126(Pt 23): 5490-5499. 


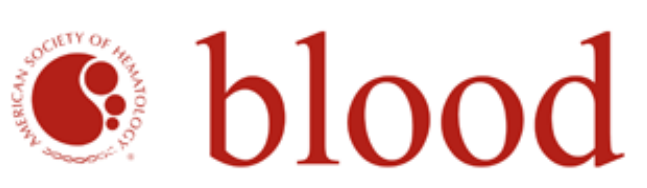

2014 123: 3185-3194

doi:10.1182/blood-2013-10-535831 originally published online April 3, 2014

\section{STXBP1 promotes Weibel-Palade body exocytosis through its interaction with the Rab27A effector Slp4-a}

Dorothee van Breevoort, Ambrosius P. Snijders, Nicola Hellen, Sarah Weckhuysen, Kathinka W. E. $M$. van Hooren, Jeroen Eikenboom, Karine Valentijn, Mar Fernandez-Borja, Berten Ceulemans, Peter De Jonghe, Jan Voorberg, Matthew Hannah, Tom Carter and Ruben Bierings

Updated information and services can be found at:

http://www.bloodjournal.org/content/123/20/3185.full.html

Articles on similar topics can be found in the following Blood collections

Thrombosis and Hemostasis (800 articles)

Vascular Biology (463 articles)

Information about reproducing this article in parts or in its entirety may be found online at:

http://www.bloodjournal.org/site/misc/rights.xhtml\#repub_requests

Information about ordering reprints may be found online at:

http://www.bloodjournal.org/site/misc/rights.xhtml\#reprints

Information about subscriptions and ASH membership may be found online at:

http://www.bloodjournal.org/site/subscriptions/index.xhtml

Blood (print ISSN 0006-4971, online ISSN 1528-0020), is published weekly by the American Society of Hematology, 2021 L St, NW, Suite 900, Washington DC 20036.

Copyright 2011 by The American Society of Hematology; all rights reserved. 